\title{
An Unusual and Malignant Intussusception in Children
}

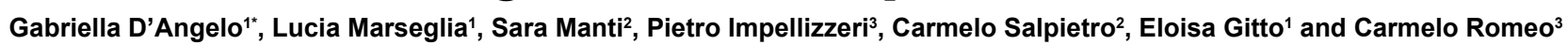

${ }^{1}$ Neonatal and Pediatric Intensive Care Unit, Department of Pediatrics, University of Messina, Italy

${ }^{2}$ Unit of Paediatric Genetics and Immunology, Department of Paediatrics, University of Messina, Italy

${ }^{3}$ Unit of Paediatric Surgery, Department of Paediatrics, University of Messina, Italy

\begin{abstract}
Renal artery embolism (RAE) is a rare disease, and its clinical features and diagnostic tools are mysterious to most physicians. Anticoagulants, surgery, and thrombolytic therapies have been used to treat patients with RAE. However, there is no universal protocol for the proper management of RAE and the timing of treatment. This variation in treatment management further impairs the comparison of different therapies, complications and prognoses.

We reported a RAE patient who underwent intra-arterial urokinase treatment. A detailed literature search found that the most common presentations of RAE are localized pain in the flank/abdomen, nausea and vomiting, and fever. A few laboratory abnormalities, including elevations of lactic dehydrogenase, C-reactive protein, and white cell count, as well as unexplained proteinuria and hematuria, are useful screening tools for RAE. A contrast-enhanced computed tomographic scan of the abdomen is currently the best diagnostic tool. Anticoagulants are an effective and safe treatment, resulting in a fair prognosis for RAE cases. The rates of mortality and long-term hemodialysis are low. Surgery and intra-arterial thrombolytic therapy should be reserved in cases where the aggressive preservation of residual renal function is necessary in patients with deteriorated renal function or only one functional kidney. In addition, concurrent and subsequent thrombolytic events in other organs are common in patients with RAE.
\end{abstract}

Keywords: Malignant tumour; Small intestine; Bowel obstruction; Surgery; Child

\section{Case History}

An 8-year-old boy presented with previous medical history of abdominal pain and nausea with vomiting following meals. His symptoms progressively worsened to severe anorexia, weight loss, constipation, and bilious vomiting on the day of admission. Two months earlier the patient had similar symptoms. His vital signs on admission were within normal limits. He had generalized tenderness without peritoneal signs. No palpable mass was identified. Bowel sounds were exaggerated. Digital rectal examination was normal. He did not present fever, chills, bleeding per rectum, or previous abdominal surgeries. His laboratory investigations only showed increased C-reactive protein ( $2.5 \mathrm{mg} / \mathrm{L}$, normal range $0-0.5 \mathrm{mg} / \mathrm{L})$. Liver function tests were normal. Abdominal X-ray revealed no specific bowel gas pattern with gaseous distention of several small bowel loops. Focused sonography of the right lower quadrant reported a "target sign" or "doughnut", pseudo kidney/ sandwich appearance (Figure 1), suggesting bowel intussusception. This abdominal ultrasonography imaging has a high sensitivity and specificity in its detection and it is usually not mistaken for other intestinal diseases.

Abdominal CT revealed a typical target-appearing lesion, extending for approximately $16 \mathrm{~cm}$ of the terminal ileum and ascending colon into the transverse colon. Liver metastasis was also described. Emergency laparotomy confirmed a distal ileo-colic intussusception. The laparotomy was performed and was noted a ileo-colic intussusception with a $4 \mathrm{~cm} \times 4 \mathrm{~cm}$ intraluminal growth in the lumen, large and hard mesenteric mass. Were also detected enlarged mesenteric lymphnodes (Figure 2). The involved segment presented edema and initial necrosis with loss of the classic three-layer intestinal mucosa. It was resected with $5 \mathrm{~cm}$ margins and end to end anastomosis with healthy small bowel was performed before treating intussusception, one dose of cefoxitin $(40 \mathrm{mg} / \mathrm{kg})$ has been administered.

The resection was complete with viable ends. Postoperatively, the patient made an uneventful recovery and was allowed oral clear fluids on five postoperative day and semisolid diet on the seventh postoperative day. The patient in the follow-up period underwent contrast enhanced CT scan of the thorax, abdomen and pelvis to look

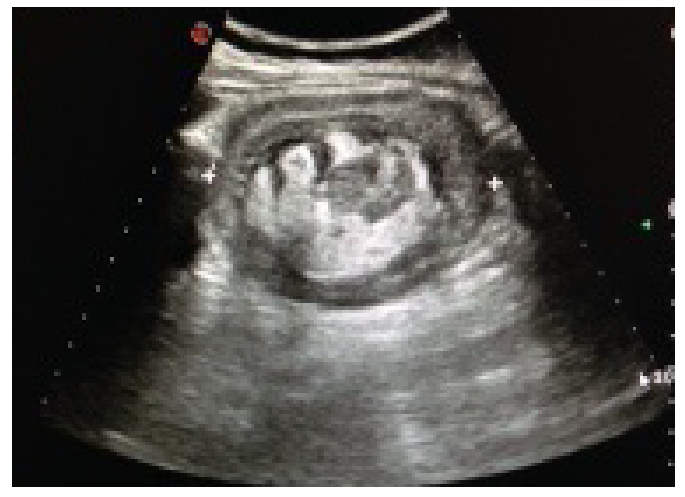

Figure 1: Abdominal ultrasound noted an intussusception, giving the typical "target" sign.

for synchronous lesions detected at the admission (hepatic lesions). Therefore, the child was thereafter referred to the Medical Oncology and Radiation Oncology Department where he is being considered for adjuvant chemotherapy and is under fortnightly follow-up.

The leading point of the invagination was a diffuse large B-cell lymphoma (DLBCL), measuring $4 \times 4 \mathrm{~cm}$, consisting of centroblastic/ polymorphic cells of intermediate-large size; $\mathrm{CD} 20+, \mathrm{CD} 10+, \mathrm{bcl}^{2}-$, bcl6+, CD5-, D1, CKAE1-AE3, AML- MIB > 70\%. All nodes showed strangles reactive hyperplastic follicle-sinus histiocytosis and lymphostasis.

Intussusception is often seen in infants and children; it can be

*Corresponding author: Gabriella D'Angelo, Department of Pediatrics, University of Messina, Via Consolare Valeria, 1, 98125 Messina, Italy, Tel: +390902213100 Fax: +390902213876; E-mail: gabridangelo@alice.it

Received April 13, 2016; Accepted May 02, 2016; Published May 09, 2016

Citation: D'Angelo G, Marseglia L, Manti S, Impellizzeri P, Salpietro C, et al. (2016) An Unusual and Malignant Intussusception in Children. Gen Med (Los Angel) 4 248. doi:10.4172/2327-5146.1000248

Copyright: (c 2016 D'Angelo G, et al. This is an open-access article distributed under the terms of the Creative Commons Attribution License, which permits unrestricted use, distribution, and reproduction in any medium, provided the original author and source are credited. 


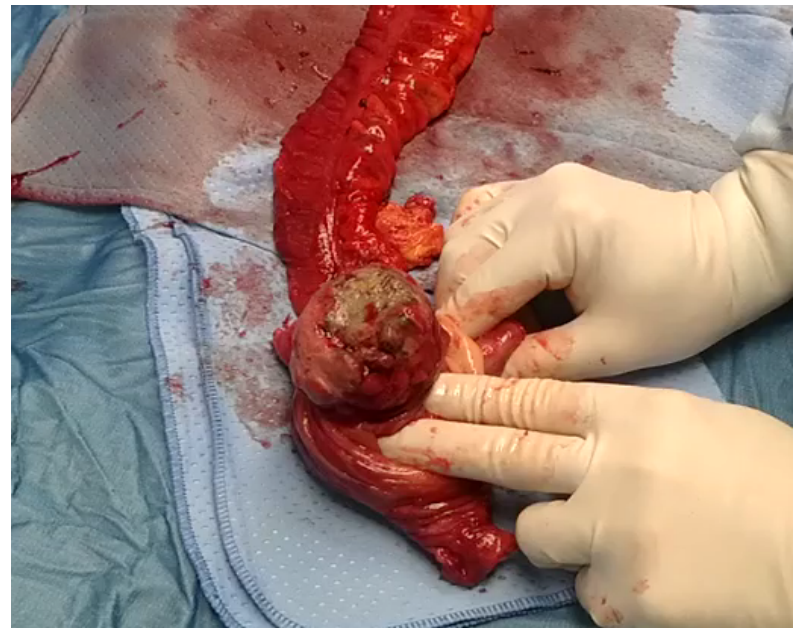

Figure 2: Resected specimen showing the large B-cell lymphoma.

transient and occasionally a spontaneous resolution is reported. Also in our case, the recurrence of clinical symptoms, as abdominal pain and vomiting, could suggest a self-limiting intussusception.

Intussusception occurring in the large bowel is more likely to be related to malignant lesions in $63 \%-68 \%$ of cases [1]. Primary malignant tumours of the small intestine are very rare, accounting for less than $2 \%$ of all gastrointestinal malignancies [2]. Lymphoma constitutes $15 \%-20 \%$ of all small intestine neoplasms and $20 \%-30 \%$ of all primary gastrointestinal lymphomas [3]. Especially, diffuse large B-cell lymphoma (DLBCL), the most common form of nonHodgkin lymphoma, is a heterogeneous entity encompassing a range of clinical and morphological features [4]. Non-Hodgkin lymphoma accounts for $40-50 \%$ of all lymphomas in children and adolescents. It represents approximately $6 \%$ of all malignancies in children [5]. It is a heterogeneous clinical entity, rarely causing acute obstructive symptoms and intussusceptions [4,6]. Ileum is the most common site $(60 \%-65 \%)$, because of its major concentration of gut-associated lymphoid tissue, followed by jejunum $(20 \%-25 \%)$, duodenum $(6 \%$ - $8 \%)$ and other sites $(8 \%-9 \%)$ [7]. They may be solitary or diffuse; solitary form tends to encircle the bowel and narrow the lumen, diffuse form shows multi-segment involvement with numerous polypoidal excrescences. Intestinal involvement by NHL was correlated to increased frequency of abdominal symptoms resulting in an earlier diagnosis. A mesenteric or retroperitoneal mass that does not involve the bowel wall and it remains clinically silent until. It was found that the invasion depth of the tumor is significantly associated with patient survival [8]. DLBCL-patients initially undergo chemotherapy rather than surgery. Therefore, the relationship between surgery treatment and prognosis has not previously been reported. The importance of resection of bowel containing any slightest lesion, along with removal of the regional lymph nodes, is further stressed. However, a multicenter study found that primary surgical resection was associated with a favourable prognosis in cases of intestinal DLBCL, encouraging surgical resection as primary treatment [9]. Conversely, it has been also reported an increased risk of gastric adenocarcinoma after treatment of primary gastric lymphoma, especially of diffuse large B-cell lymphoma [10]. Finally, there is no consensus on the optimal treatment against primary gastrointestinal DLBC [3].

In conclusion, we describe a case of large B-cell lymphoma causing an ileo-colic intussusception in children. In cases of intussusception, especially in the older age group of the children, we need to keep a high index of suspicion for malignant lymphoma of the bowel, including DLBCL.

\section{Acknowledgement} article.

The authors are grateful to the patient and his family for their support for our

\section{References}

1. Akbulut S (2012) Unusual cause of adult intussusception: diffuse large B-cell non-Hodgkin's lymphoma: a case report and review. European Review for Medical and Pharmacological Sciences 16: 1938-1946.

2. Xu XQ, Hong T, Li BL, Liu W (2013) Ileo-ileal intussusception caused by diffuse large B-cell lymphoma of the ileum. World Journal Gastroenteroly 19: 84498452.

3. Ghimire P, Wu GY, Zhu L (2011) Primary gastrointestinal lymphoma. World Journal Gastroenteroly 17: 697-707.

4. Stein H, Warnke RA, Chan WC (2008) Diffuse large B-cell lymphoma, not otherwise specified. In: Swerdlow SH, Campo E, Harris NL (eds.) WHO classification of tumours of haematopoietic and lymphoid tissues (4th edn) Lyon, France: IARC, pp: 233-237.

5. Linabery AM, Ross JA (2008) Childhood and adolescent cancer survival in the US by race and ethnicity for the diagnostic period 1975-1999. Cancer 113: 2575-2596.

6. Li B, Shi YK, He XH (2008) Primary non-Hodgkin lymphomas in the small and large intestine: clinico-pathological characteristics and management of 40 patients. International Journal of Hematology 87: 375-381.

7. Schottenfeld D, Beebe-Dimmer JL, Vigneau FD (2009) The epidemiology and pathogenesis of neoplasia in the small intestine. Annals of Epidemiology 19 : $58-69$.

8. Simpson T, Ivey J, Borkowski S (2004) Pediatric management problems Intussusception. Pediatric Nursing 30: 326-327.

9. Kim SJ, Kang HJ, Kim JS (2011) Comparison of treatment strategies for patients with intestinal diffuse large B-cell lymphoma: surgical resection followed by chemotherapy versus chemotherapy alone. Blood 117: 1958.

10. Inaba K, Kushima R, Murakami N, Kuroda Y, Harada K, et al. (2013) Increased risk of gastric adenocarcinoma after treatment of primary gastric diffuse large B-cell lymphoma. BMC Cancer 13: 499. 